\title{
A liver analog construct for use as an alcoholic liver disease model
}

\author{
LIU HaiXia ${ }^{1 *}$, LI ShengJie ${ }^{2}$, YAN YongNian ${ }^{2}$, WANG XiaoHong ${ }^{2}$, LIN Feng $^{2} \&$ ZHANG RenJi ${ }^{2}$ \\ ${ }^{1}$ School of Instrument Science and Opto-electronic Engineering, Beihang University, Beijing 100191, China; \\ ${ }^{2}$ Department of Mechanical Engineering, Tsinghua University, Beijing 100084, China
}

Received August 30, 2010; accepted March 11, 2011

\begin{abstract}
The construction of an in vitro liver analog is likely to be of great use for the study of alcoholic liver disease (ALD) and other hepatic disorders. Preliminary results indicate that multi-cell assembling technology can produce two hybrid cell-matrix spatial distributions according to predesigned digital models; therefore, it is possible to fabricate a liver-like construct that can mimic the structure and main components of the natural liver. After in vitro culture, a liver analog was formed. Employing the rat liver analog constructs cultured in vitro for several days for the study of ALD by adding alcohol, MDA and NO concentration were altered and controlled by adding vitamin E and vitamin C. ALD is considered an important factor in liver cancer where the pathogenesis is complex but the mechanism is still not well understood. Previous research of pathogenesis and drug prevention in ALD was based on animal models, such as baboons or rats, with particular limitations. Therefore, multi-cell assembling technology used to construct human liver analogs that contain various human liver cells is likely to be useful for studying ALD pathogenesis and related drug research.
\end{abstract}

multi-cell assembling, liver analog construct, alcoholic liver disease

Citation: $\quad$ Liu H X, Li S J, Yan Y N, et al. A liver analog construct for use as an alcoholic liver disease model. Chin Sci Bull, 2012, 57: 955-958, doi: 10.1007/s11434-012-4976-7

The liver is an important organ with many functions that involve metabolism, synthesis, regulation, and detoxification. Importantly, over $95 \%$ of alcohol intake by humans is metabolized by the liver. Alcoholic liver disease (ALD) is one of the major causes of morbidity and mortality both in the United States and worldwide. The mortality of cirrhosis with superimposed alcoholic hepatitis is worse than that of many common types of cancers [1,2]. Recent studies have revealed that alcoholic hepatitis has become an important health problem facing the developed and developing world.

The mechanism of ALD is complex and the pathogenesis is still not well understood. At present, basic research on pathogenesis and drug prevention is based on animal models such as baboons or rats [3]. However, this application is limited because of animal requirements and high costs, and commonly seen as undesirable because of ethical considerations [4,5]. A liver analog based on three-dimensional (3D)

*Corresponding author (email: liuhx02@mails.tsinghua.edu.cn) cell assembling technology has some similar liver functions to those found in the physiological environment $[6,7]$. The current study describes multi-cell assembling technology [8] using liver cells and adipose-derived stromal cells (ADSCs) to build liver analogs with vascular access. Logistical issues such as selecting the appropriate extracellular matrix material, culture conditions in vitro and biological evaluation, were investigated for the application of this technology as an ALD model.

Previous studies [9] have shown that the metabolism of alcohol by the liver results in the formation of a malondialdehyde (MDA) as a product of increased lipid peroxidation. Therefore, the level of MDA indirectly reflects the degree of liver injury. MDA has also been shown to impair the normal function of endothelial cell distribution in the liver vascular network; the content of vascular endothelial diastolic factor, nitric oxide (NO), reflects normal endothelial cell function [10].

Vitamins $\mathrm{C}$ and $\mathrm{E}$ are powerful antioxidants and it has 
been shown that their combined application improves the proliferation of liver cells, and protects the detoxification ability of the liver and normal metabolism [11]. By adding alcohol to stimulate liver analog constructs fabricated by multi-cell assembling technology during in vitro culturing, MDA and NO concentrations were measured by adding vitamin $\mathrm{E}$ and vitamin $\mathrm{C}$ [12].

The liver is a highly vascular system composed of two branched vascular networks and numerous hepatic lobules that are important for liver function [13]. By constructing a 3D structure containing an artificial vascular system we were able to simulate the liver structure, thereby constructing a suitable liver analog. Liver cells and matrix materials, such as gelatin (Tianjin Green-Island Company, China, medical level), chitosan (Sigma, USA), and sodium alginate (Sigma, USA) (G/C/A system) were used to construct a liver lobular structure. Adipose-derived stromal cells (ADSCs) and a gelatin, fibrin (Sigma, USA), and sodium alginate (G/F/A system) solution was used to produce the vascular channels.

Specialized software was used to design a heterogeneous computer-aided design (CAD) model to mimic the organizational structure of the liver (Figure 1). Materials were calibrated for the component in accordance with the model structure using Aurora preprocessing software (Yinhua, China), and the digital model was transferred to a common layer interface (CLI) file for digital-controlled assembly.

Liver cells were isolated from 6-week-old Sprague-Dawley (SD) rats (Laboratory Animal Center, Health Science Center, Peking University, China) using a previously established procedure involving a two-step collagenase (Invitrogen, USA) perfusion method [14,15]. Approximately $96 \%$ of cells were identified as typical hepatocytes, while the remainders were non-parenchymal cells. ADSCs were isolated from the inguinal adipose tissue of SD rats, as previously described, and were then differentiated into endothelial cells after $4 \mathrm{~d}$ induction. The $15 \mathrm{G} 0.5 \mathrm{C} 1.25 \mathrm{~A}$ and $15 \mathrm{G} 2.5 \mathrm{~F}-$ $1.25 \mathrm{~A}$ sol materials prepared for visually distinguishing the corresponding solvents are phosphate-buffered saline (PBS) solution and DMEM/F12 medium (Invitrogen, USA). Both

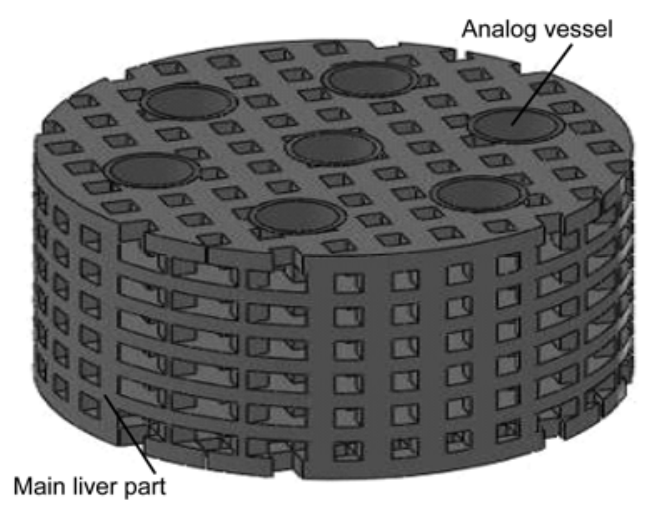

Figure 1 Liver analog computer-aided design (CAD) model. sols were gently mixed (being sure to avoid the introduction of air bubbles) with the liver cells at $5 \times 10^{6}$ cells $/ \mathrm{mL}$ and endothelial cells induced by ADSCs at $1 \times 10^{6}$ cells $/ \mathrm{mL}$. Cells were loaded into standard medical disposable sterile syringes (Shanghai Kangshou, $1 \mathrm{~mL}$ ) with a 26-gauge needle (OD: 0.45 , ID: $0.25, \mathrm{~L}: 10 \mathrm{~mm}$ ) and fitted to the Multi-nozzle Cell Assembler [8]. Figure 2 shows a formed 3D liver analog construct. The red tube-like component was made of ADSC/GFA hydrogel, where the red color due to the solvent of GFA was DMEM/F12, while the white meshwork was made of hepatocytes/GCA hydrogel. This complex structure with vascular-like network was similar to the designed digital model (Figure 1).

The fabricated construct was removed from the Multinozzle Cell Assembler working chamber and a posttreatment was conducted [16]. The assembly was statically cultured at $37^{\circ} \mathrm{C}, 5 \% \mathrm{CO}_{2}$ in a $\mathrm{MCO}-15 \mathrm{AC}$ incubator (Sanyo, Japan) with DMEM medium supplemented with $15 \%$ fetal bovine serum (FBS), $100 \mathrm{IU} / \mathrm{mL}$ penicillin/streptomycin, and $1 \mathrm{IU} / \mathrm{mL}$ aprotinin (Sigma, USA). Culture medium was changed every other day. During culture, the morphology and proliferation of cells in the construct were observed daily using an inverted microscope (CK40; Olympus, Japan).

The construct was cultured for 10 days and the distribution and growth status of all cells was observed by immunohistochemical staining. The construct was rinsed with PBS, fixed with $4 \%$ (w/v) paraformaldehyde (Tianjing Keou, China) at $48^{\circ} \mathrm{C}$ for $2 \mathrm{~h}$, followed by several washes in distilled water, embedded in optimal cutting temperature (TissueTek O.C.T) medium, and then sectioned into $10-\mu \mathrm{m}$ sections using a HM525 freezing microtome (MICROM, Germany). Some sections were stained with $50 \mathrm{mg} / \mathrm{mL}$ propidium iodide (PI) (Sigma, USA), followed by immunofluorescence with an anti-CD3 primary antibody (goat anti-rat polyclonal at 1:200 in PBS; Santa Cruz, USA) and secondary antibody FITC-conjugated anti-goat IgG (Sigma, USA) at 1:100 in PBS. Sections were observed by fluorescence

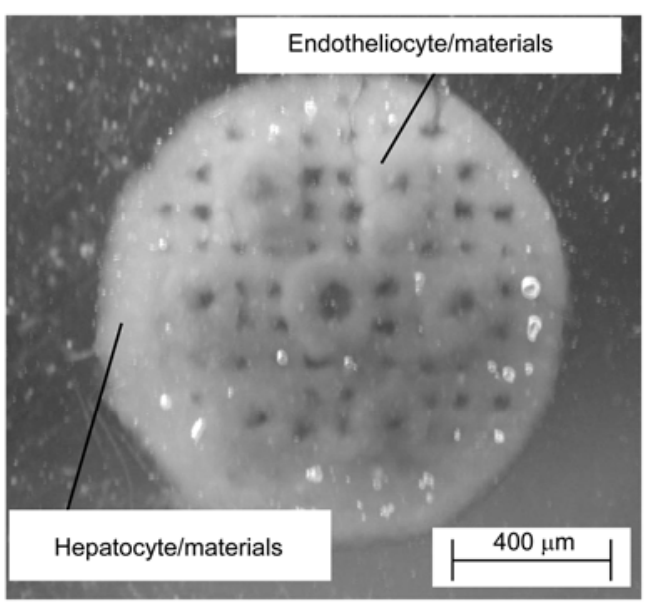

Figure 2 Liver analog construct. 
microscopy (X-Cite series 150; EXFO, Canada). The rest of the samples were stained with $50 \mathrm{mg} / \mathrm{mL}$ PI at $48^{\circ} \mathrm{C}$ for 30 min, then extensively washed with PBS, and incubated overnight at $48^{\circ} \mathrm{C}$ with primary antibody albumin (ALB) (rabbit anti-rat, monoclonal, Santa Cruz, USA, 1:200, PBS). After being washed with PBS, the samples were incubated with a secondary antibody, FITC-conjugated anti-rabbit IgG at $1: 100$ in PBS (Sigma, USA) at $37^{\circ} \mathrm{C}$ for $30 \mathrm{~min}$.

In Figure 3, PI-ALB immunofluorescence staining was positive for the nuclei, which is a distinct biomarker for mature hepatocytes. This confirmed that the hepatocytes were alive with secretion ability.

As shown in Figure 4, cells were stained with PI-CD31, a specific biomarker for mature endothelial cells. This suggested that the endothelial cells induced by ADSC were viable with the competent secretion ability and were distributed within the vascular structure.

After $10 \mathrm{~d}$ culture, the system showed an assembled construct with a vascular-like network that provided a suitable environment for the proliferation and maintenance of normal metabolic functions of both hepatocytes and endothelial cells induced by ADSC. This suggests that the system has

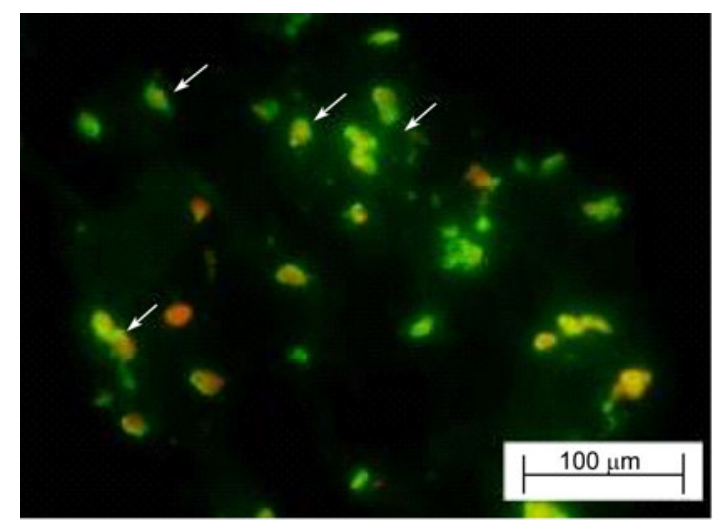

Figure 3 Immunofluorescence staining pictures of hepatocytes using anti-ALB antibodies. Arrows indicate nucleus.

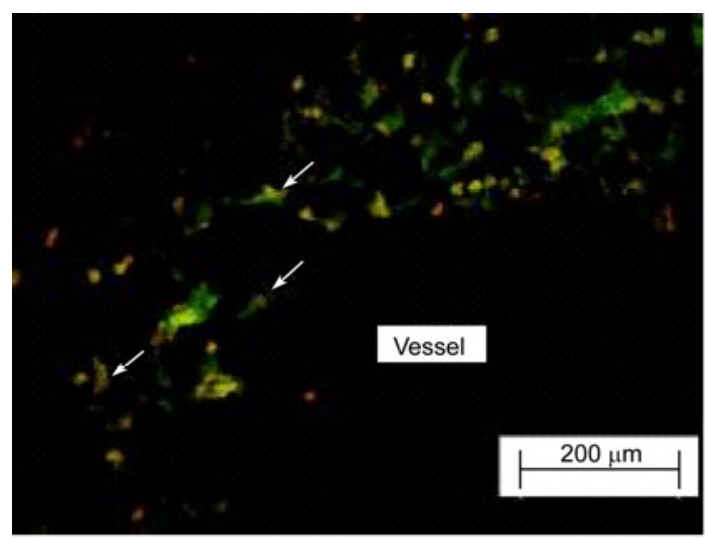

Figure 4 Immunofluorescence staining of endothelial cells using an antiCD31 antibody. Arrows indicate nucleus. the potential to be a functional liver analog. After this time, the residual liver analogs were randomly divided into two sets, namely the alcohol set and drug set.

(1) For the alcohol set, the liver analog was cultured with DMEM/F12 medium supplemented with $15 \%$ FBS, 50 $\mathrm{mmol} / \mathrm{L}$ alcohol (Beijing Yili, China). After $12 \mathrm{~h}$ culture, the medium was changed with normal DMEM/F12 with $15 \% \mathrm{FBS}, 100 \mathrm{IU} / \mathrm{mL}$ penicillin/streptomycin. The entire removed supernatant medium was preserved at $-20^{\circ} \mathrm{C}$ for further tests.

(2) For the drug set, the liver analog was cultured in DMEM medium supplemented with $15 \% \mathrm{FBS}, 50 \mathrm{mmol} / \mathrm{L}$ alcohol (Beijing Yili, China), $2.3 \mu \mathrm{mol} / \mathrm{L}$ vitamin E, and 2.3 $\mu \mathrm{mol} / \mathrm{L}$ vitamin $\mathrm{C}$ (Sigma, USA). After $12 \mathrm{~h}$ culture, the medium was changed to DMEM/F12 containing $15 \%$ FBS and $100 \mathrm{IU} / \mathrm{mL}$ penicillin/streptomycin. The removed cellfree medium was preserved at $-20^{\circ} \mathrm{C}$ for further tests.

(3) Each test group was given either the alcohol or drug every two days and sampled. The regular reserved culture media were analyzed using an automatic biochemical analyzer (7600; Hitachi, Japan). Each group of tests consisted of five samples and the data were presented as mean $\pm \mathrm{SD}$ values. MDA content was tested by the modified thiobarbituric acid (TBA) method at the absorption peak of 532-535 $\mathrm{nm}$ [17]. NO content was tested using the nitric oxide test box (Nanjing Jiancheng).

The MDA test results after $8 \mathrm{~d}$ culture are shown in Figure 5. The MDA concentration increased at the early stages and then decreased in the alcohol set. The changes in MDA concentration may be due to irreversible hepatocyte damage by the alcohol, thus, the MDA concentration gradually decreased. Comparatively, in the drug set with vitamin $\mathrm{E}$ and vitamin $\mathrm{C}$, MDA concentration changed negligibly during the test period.

The NO test results after $6 \mathrm{~d}$ in culture are shown in Figure 6 . These results suggest that by adding vitamin $\mathrm{E}$ and vitamin $\mathrm{C}$ to the pure alcohol set resulted in a slightly increased NO content. This indicated that the presence of both vitamin $\mathrm{E}$ and vitamin $\mathrm{C}$ plays protective role on endothelial cells.

Preliminary experimental results indicated that the multi-

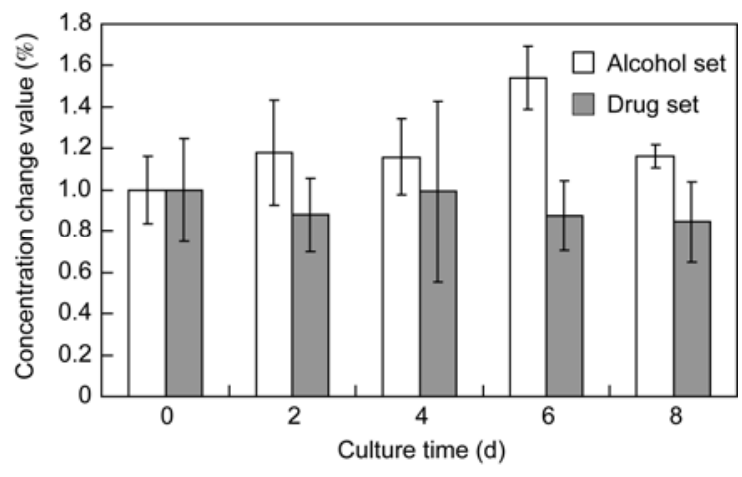

Figure 5 The changes of MDA concentration during $8 \mathrm{~d}$ culture. 


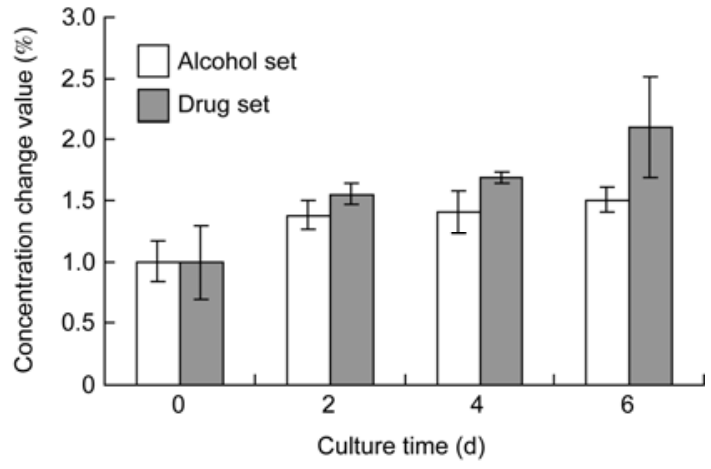

Figure 6 The changes of NO concentration during $6 \mathrm{~d}$ culture.

cell assembling technology can produce two cell-hydrogel spatial distributions according to predesigned digital models, thereby fabricating liver-like constructs that mimic the structure and main components of the natural liver. Additionally, after a period of time, the technology can form liver analogs. These liver analog constructs can be useful in alcoholic liver disease research. There is a need for further research on the system to perfect certain tests and establish technological processes. This system has great potential for use in researching human ALD pathogenesis because it can be an alternative to animal models and will likely be beneficial for studying ALD pathogenesis and related drug research.

This work was supported by the National Natural Science Foundation of China (50905009).

1 Barve A, Khan R, Marsano L, et al. Treatment of alcoholic liver disease. An Hepatol, 2008, 7: 5-15

2 McClain C J, Song Z, Barve S S, et al. Recent advances in ALD. IV. Dysregulated cytokine metabolism in ALD. Am J Physiol Gastrointestinal Liver Physiol, 2004, 287: 497-502
3 Stewart S, Jones D, Day C P. Alcoholic liver disease: New insights into mechanisms and preventative strategies. Trends in Mol Med, 2001, 7: 408-413

4 Song Z, McClain C J, Chen T. S-Adenosylmethionine protects against acetaminophen-induced hepatotoxicity in mice. Pharmacol, 2004, 71: 199-208

5 Ji C, Kaplowitz N. Betaine decreases hyperhomocysteinemia, endoplasmic reticulum stress, and liver injury in alcohol-fed mice. Gastroenterology, 2003, 124: 1488-1499

6 Liu H X, Yan Y N, Wang X H, et al. Construct hepatic analog by cell-matrix controlled assembly technology. Chin Sci Bull, 2006, 51: 1830-1835

7 Wang X H, Yan Y N, Pan Y Q, et al. Generation of three-dimensional hepatocyte/gelatin structures with rapid prototyping system. Tissue Eng, 2006, 12: 83-90

8 Li S J, Xiong Z, Wang X H, et al. Direct fabrication of a hybrid cell/hydrogel construct by a double-nozzle assembling technology. J Bioact Compat Pol, 2009, 24: 235-248

9 Mezey E, Potter J J, Rennie-Tankersley L, et al. A randomized placebo controlled trial of vitamin E for alcoholic hepatitis. J Hepatol, 2004, 40: 40-46

10 Ganaraja B, Crystal D D S, Vijayalakshmi B M, et al. Use of vitamin $\mathrm{C}$ on effect of ethanol induced lipid peroxidation in various tissues, sperm count \& morphology in the wistar rats. J Chin Clin Med, 2008, 3: $627-631$

11 Oyinbo C A, Dare W N, Okogun G R A, et al. The hepatoprotective effect of vitamin $\mathrm{C}$ and $\mathrm{E}$ on hepatotoxicity induced by ethanol in sprague dawley rats. Pakistan J Nutr, 2006, 5: 507-511

12 Tantcheva L P, Stoeva E S, Galabov A S, et al. Effect of vitaminamin $\mathrm{E}$ and vitaminamin $\mathrm{C}$ combination on experimental influenza virus infection. Methods Find Exp Clin Pharmacol, 2003, 25: 259-264

13 Luong E, Gerecht S. Stem cells and scaffolds for vascularizing engineered tissue constructs. Adv Biochem Eng Biotechnol, 2009, 114: 129-172

14 Pei H Y, Wang Y F, Pei X T. Differentiation of human embryonic stem cells along a hepatocyte lineage and its application in liver regeneration. Chin Sci Bull, 2008, 53: 1-7

15 Papeleu P, Vanhaecke T, Henkens T, et al. Isolation of Rat Hepatocytes. 3rd ed. Totowa: Humana Press, 2006

16 Liu H X, Yan Y N, Wang X H. Structure stability research of cells assembly in vitro culturing (in Chinese). J Tsinghua Univ, 2010, 50: 1197-1200

17 Tokur B, Korkmaz K, Ayas D. Comparison of two thiobarbituric acid (TBA) method for monitoring lipid oxidation in fish. J Fish Aquat Sci, 2006, 23: 331-334

Open Access This article is distributed under the terms of the Creative Commons Attribution License which permits any use, distribution, and reproduction in any medium, provided the original author(s) and source are credited. 